\title{
Connectivity of Circulant Digraphs
}

Erik A. van Doorn

DEPARTMENT OF APPLIED MATHEMATICS

TWENTE UNIVERSITY OF TECHNOLOGY

P.O. BOX 217

7500 AE ENSCHEDE,

THE NETHERLANDS

\section{ABSTRACT}

An explicit expression is derived for the connectivity of circulant digraphs.

\section{INTRODUCTION}

A digraph $D$ consists of a finite set of vertices $V(D)$ and a set of $\operatorname{arcs} A(D)$ which is a subset of all ordered pairs $(u, v), u, v \in V(D)$, and $u \neq v$. If $(u, v) \in A(D)$, then $u$ is a predecessor of $v$ and $v$ is a successor of $u$.

For a subset $U \subset V(D), D-U$ denotes the subgraph of $D$ induced by $V(D)-U$. Furthermore, the set

$$
\mathcal{O}_{D}(U)=\{v \in V(D)-U:(u, v) \in A(D) \text { for some } u \in U\}
$$

is called the outset of $U$ in $D$. If $U=\{u\}$ we write $O_{D}(u)$ instead of $O_{D}(\{u\})$. Also, if $E$ is a subgraph of $D$ we write $\mathcal{O}_{D}(E)$ instead of $\mathcal{O}_{D}(V(E))$.

A cut-set $C$ of $D$ is a proper subset of $V(D)$ such that $D-C$ either is not strongly connected or consists of a single vertex. $C$ is a minimum cut-set if any other cut-set of $D$ has at least $|C|$ vertices. The size of a minimum cut-set is denoted by $\kappa(D)$ and called the connectivity of $D$. Clearly, $\kappa(D) \leq|V(D)|-1$ with equality subsisting only if $D$ is complete.

For a fixed integer $n>0$ and set $S \subset\{1,2, \ldots, n-1\}$ we construct a digraph $D=D(n, S)$ with vertices $v_{0}, v_{1}, \ldots, v_{n-1}$ as follows: $\left(v_{i}, v_{j}\right) \in A(D)$ if and only if $j-i \equiv s(\bmod n)$ for some $s \in S$. Any digraph that can be constructed in the above manner is called a circulant digraph (or directed star polygon). In this note we derive an explicit expression for the connectivity $\kappa(D)$ of a circulant digraph $D=D(n, S)$ in terms of its order $n$ and its symbol $S$. 
For any vertex $v \in V(D)$ of a circulant digraph $D=D(n, S)$ one has

$$
\left|\mathcal{O}_{D}(v)\right|=|S|,
$$

so that an upperbound for the connectivity of $D$ is given by

$$
\kappa(D) \leq|S|,
$$

since removal of $O_{D}(v)$ from $D$ disrupts all directed paths from $v$ to any other vertex in $D-O_{D}(v)$. If equality holds in (2) the circulant digraph $D$ is said to be $\kappa$-optimal. It is of interest in network design studies to be able to identify the class of circulant digraphs which are $\kappa$-optimal. Our basic result on the connectivity of circulant digraphs, which is stated in Section 3, enables us to perform this identification. It will subsequently be shown that the known sufficient conditions for $\kappa$-optimality of circulant digraphs follow readily from our result. Section 2 contains some preliminary results.

If the symbol of a circulant digraph $D=D(n, S)$ is such that $s \in S<=>$ $n-s \in S$, so that the $\operatorname{arc}\left(v_{i}, v_{j}\right) \in A(D)$ if and only if $\left(v_{j}, v_{i}\right) \in A(D)$, we can replace $D(n, S)$ by the corresponding graph $G(n, S)$ in which $v_{i}$ is adjacent to $v_{j}$ if and only if the pair of arcs $\left(v_{i}, v_{j}\right)$ and $\left(v_{j}, v_{i}\right)$ are in $A(D)$. Any graph that can be obtained in this way is a circulant graph. Defining connectivity for graphs in the usual way [7], a circulant graph and its corresponding digraph have the same connectivity. The recently obtained necessary and sufficient condition for $\kappa$-optimality of circulant graphs [4] and all previously known sufficient conditions (see the references in [4]) can therefore be obtained from our result by thinking of such a graph as the corresponding digraph.

In what follows circulant stands for circulant digraph. When we consider labeled circulants it is always tacitly understood that the labeling corresponds to the construction rule for circulants. For concepts used but not defined here we refer to Harary's book [7].

\section{MODULES AND ATOMIC TRAPS}

We consider a circulant $D=D(n, S)$ with vertices $v_{0}, v_{1}, \ldots, v_{n-1}$. Let $r$ be a divisor of $n$ (notation: $r \mid n$ ). For $i=0,1, \ldots, r-1$ we denote by $F_{r}^{i}=F_{r}^{i}(D)$ the subgraph of $D$ which is induced by the set of vertices $V\left(F_{r}^{i}\right)=$ $\left\{v_{i}, v_{i+r}, v_{i+2 r}, \ldots, v_{i+n-r}\right\}$. Any such subgraph $F_{r}^{i}$ will be called a module of $D$ (of order $n / r$ ). We next define

$$
M(r, S)=\{l: 1 \leq l<r \text { and } l \equiv s(\bmod r) \text { for some } s \in S\},
$$

so that, in particular, $M(n, S)=S$ and $M(1, S)=\emptyset$.

Lemma 1. For any $r \mid n$ and $i \in\{0,1, \ldots, r-1\}$ one has 


$$
\left|\mathscr{O}_{D}\left(F_{r}^{i}\right)\right|=\frac{n}{r}|M(r, S)|
$$

Proof. It is clear that if $V\left(F_{r}^{j}\right)$ contains a successor of a vertex in $V\left(F_{r}^{i}\right), i \neq j$, then each vertex of $V\left(F_{r}^{j}\right)$ is a successor of a vertex in $V\left(F_{r}^{i}\right)$. Hence, $\left|\mathcal{O}_{D}\left(F_{r}^{i}\right)\right|$ is $n / r$ times the number of modules $F_{r}^{j}, j \neq i$, which contain successors of a vertex in $F_{r}^{i}$. But then, one has $\left(v_{i+k r}, v_{j+r r}\right) \in A(D)$ for some $k$ and $l(0 \leq k, l<n / r)$ if and only if either $j>i$ and $j-i \in M(r, S)$ or $j<i$ and $r+j-i \in$ $M(r, S)$, whence this number of modules equals $|M(r, S)|$.

If $\left|\mathcal{O}_{D}\left(F_{r}^{i}\right)\right|<n-n / r$, or, equivalently, $|M(r, S)|<r-1$, then $\mathscr{O}_{D}\left(F_{r}^{i}\right)$ constitutes a cut-set. We can therefore state the following.

Lemma 2. For each $r \mid n$ such that $|M(r, S)|<r-1$ one has

$$
\kappa(D) \leq \frac{n}{r}|M(r, S)| .
$$

In what follows we will show that equality holds in (5) for some $r \mid n$. The main obstacle in establishing this result is a proof for the fact that $D$ contains a minimum cut-set which is the outset of a module of $D$. The remainder of this section will be dedicated to this task. We start off with some terminology.

The strongly connected components of the subgraph $D-C$ of a digraph $D$, where $C$ is a minimum cut-set of $D$, are called parts of $D$ with respect to $C$. A part $T$ of $D$ with respect to $C$ whose vertices have no successors outside $T$ in the subgraph $D-C$ will be called a trap of $D$ with respect to $C$. Clearly, for each minimum cut-set of $D$ there is at least one trap. Furthermore, it is readily verified that if $T$ is a trap of $D$ with respect to the minimum cut-set $C$, then $C$ consists precisely of the successors of the vertices in $T$ which are outside $T$, that is, $C=\mathscr{O}_{D}(T)$. A trap $T$ of $D$ with the property that $|V(T)|$ does not exceed the order of any other trap of $D$ is an atomic trap. The order of an atomic trap will be denoted by $a(D)$.

We will investigate the structure of an atomic trap of a circulant. It will be convenient, however, to first consider self-converse digraphs, that is, digraphs $D=(V(D), A(D))$ with the property that $D$ is isomorphic to its converse $D^{\prime}=$ $\left(V\left(D^{\prime}\right), A\left(D^{\prime}\right)\right)$, where $V\left(D^{\prime}\right)=V(D)$ and $A\left(D^{\prime}\right)=\{(u, v):(v, u) \in A(D)\}$. The next lemma, which is easy to verify (cf. the proof of Proposition 3 in [1]), shows that this context is more general.

Lemma 3. A circulant is self-converse.

If $D$ is a self-converse digraph and $P$ is a part of $D$ with respect to the cut-set $C$ such that the vertices of $P$ have no predecessors outside $P$ in the subgraph $D-C$, then $P$ is a trap of $D^{\prime}$ with respect to $C$, whence $|V(P)| \geq a\left(D^{\prime}\right)=$ $a(D)$. Therefore, we can invoke Proposition 1 of [6] to conclude the following. 
Lemma 4. In a self-converse digraph distinct atomic traps are disjoint.

We are now ready to state the key result of this section.

Lemma 5. An atomic trap of a circulant $D$ is a module of $D$.

Proof. Let $V(D)=\left\{v_{0}, v_{1}, \ldots, v_{n-1}\right\}$ be the vertex set of the circulant $D$ and let $T$ be an atomic trap of $D$. If $|V(T)|=1$ then the lemma is trivially true, therefore assume $|V(T)|>1$. Since $\pi \equiv\left(v_{0} v_{1} \cdots v_{n-1}\right) \in \operatorname{Aut}(D)$, the subgraph of $D$ induced by $\pi^{i}(V(T))$ is an atomic trap of $D$ for any $i$. Moreover, by Lemmas 3 and 4, either $\pi^{i}(V(T))=\pi^{j}(V(T))$ or $\pi^{i}(V(T)) \cap \pi^{j}(V(T))=\emptyset$. Thus $\left\{\pi^{\mathrm{i}}(V(T)): i=0,1, \ldots, n-1\right\}$ is an imprimitive block system for $\langle\pi\rangle$, the group generated by $\pi$. Since blocks of $\langle\pi\rangle$ are vertex sets of modules, $T$ is a module.

\section{CONNECTIVITY OF CIRCULANTS}

By Lemma 5 an atomic trap of the circulant $D=D(n, S)$ is a module of $D$ of order $a=a(D)$. Since the minimum cut-set corresponding to an atomic trap is the outset of that atomic trap, it follows by Lemma 1 that

$$
\kappa(D)=a\left|M\left(\frac{n}{a}, S\right)\right| .
$$

If $D$ is complete, then $a=1$ and $\kappa(D)=n-1$. On the other hand, if $D$ is not complete, then removal of the minimum cut-set corresponding to an atomic trap leaves at least two parts, whence $\kappa(D)<n-a$, i.e., $|M(n / a, S)|<n / a-1$. Lemma 2 subsequently yields our main result.

Theorem 1. The connectivity $\kappa(D)$ of a circulant $D=D(n, S)$ which is not complete is given by

$$
\kappa(D)=\min \left\{\frac{n}{r}|M(r, S)|: r \mid n \text { and }|M(r, S)|<r-1\right\} .
$$

Example. The circulant $D(12,\{1,4,5,9\})$ has connectivity 3 as appears from the results of Table I.

Some corollaries to Theorem 1 will now be derived. First we will give a condition for a circulant $D$ to be strongly connected, i.e., $\kappa(D) \geq 1$, which was mentioned earlier in [2].

Corollary 1. The circulant $D(n, S)$, where $S=\left\{s_{1}, s_{2}, \ldots, s_{|S|}\right\}$ is strongly connected if and only if g.c.d. $\left(n, s_{1}, s_{2}, \ldots, s_{|S|}\right)=1$. 
TABLE I. Connectivity analysis for the circulant of order $n=12$ with symbol $S=\{1,4,5,9\}$

\begin{tabular}{rllc}
\hline \hline$r$ & $M(r, S)$ & $|M(r, S)|<r-1 ?$ & $\frac{n}{r}|M(r, S)|$ \\
\hline 1 & $\emptyset$ & no & $(0)$ \\
2 & $\{1\}$ & no & $(6)$ \\
3 & $\{1,2\}$ & no & $(8)$ \\
4 & $\{1\}$ & yes & 3 \\
6 & $\{1,3,4,5\}$ & yes & 8 \\
12 & $\{1,4,5,9\}$ & yes & 4 \\
\hline
\end{tabular}

Proof. By Theorem 1 we have $\kappa(D) \geq 1$ if and only if $|M(r, S)|>0$ for all $r \mid n, r>1$. The statement follows readily.

The most important problem pertaining to connectivity of circulants is to determine whether a given circulant $D(n, S)$ is $\kappa$-optimal. A simple sufficient condition is given in the next corollary.

Corollary 2. The circulant $D(n, S)$ is $\kappa$-optimal if g.c.d. $(n, s)=1$ for all $s \in S$.

Proof. Let $T$ be an atomic trap of $D=D(n, S)$ and assume $v_{0} \in V(T)$. If $|V(T)|=a(D)=1$, then, by $(6), \kappa(D)=|M(n, S)|=|S|$, so that $D$ is $\kappa$-optimal. Next suppose $|V(T)|>1 . T$ is strongly connected, whence there exists an $s \in S$ such that $v_{s} \in V(T)$. Since g.c.d. $(n, s)=1$ and $T$ is a module of $D$, it follows that $T=D$, which is a contradiction.

In particular, $D(n, S)$ is $\kappa$-optimal if $n$ is prime.

The next sufficient condition for $\kappa$-optimality was stated (without proof) in [5] and (with an incorrect proof) in [8]. ( $\lceil x\rceil$ denotes the smallest integer greater than or equal to $x$.)

Corollary 3. If the circulant $D(n, S)$ is such that $i \in S$ for $i=1,2, \ldots$, $\lceil|S| / 2\rceil$, then $D(n, S)$ is $\kappa$-optimal.

Proof. If $r \mid n$ and $n>r>\lceil|S| / 2\rceil$, then $|M(r, S)| \geq|S| / 2$. Since $n / r \geq 2$, it follows that $(n / r)|M(r, S)| \geq|S|$. If, on the other hand, $r \leq\lceil|S| / 2\rceil$, then $|M(r, S)|=r-1$. By Theorem 1 we therefore have $\kappa(D)=$ $|M(n, S)|=|S|$.

We finally remark that $\kappa$-optimality for the more special case where $S=\{1,2, \ldots,|S|\}$ was proven in [3]. 


\section{ACKNOWLEDGMENT}

The author wishes to thank Willem Haemers for his helpful comments and a referee for suggesting the short proof of Lemma 5.

\section{References}

[1] B. Alspach, On point-symmetric tournaments. Canad. Math. Bull. 13 (1970) 317-323.

[2] H. Ariyoshi, Feedback arc sets of directed star polygons. IEEE Conference Record of the Fourteenth Asilomar Conference on Circuits, Systems and Computers (D. E. Kirk, ed.). IEEE Computer Society, New York (1981) $55-59$.

[3] J. N. Ayoub and I. T. Frisch, Optimally invulnerable directed communication networks. IEEE Trans. Comm. Tech., vol. COM-18 (1970) 484-489.

[4] F. Boesch and R. Tindell, Circulants and their connectivities. J. Graph Theory, 8 (1984) 487-499.

[5] I. T. Frisch and M. Malek-Zavarei, Vulnerability of directed communication nets. IEEE Trans. Circuit Theory, vol. CT-19 (1972) 283-284.

[6] Y. O. Hamidoune, Sur les atomes d'un graphe orienté. C.R. Acad. Sc. Paris Sér. A 284 (1977) 1253-1256.

[7] F. Harary, Graph Theory. Addison-Wesley, Reading, MA (1969).

[8] M. Malek-Zavarei and I. T. Frisch, A class of optimally invulnerable directed star polygons. Proc. 13th Midwest Circuit Theory Symp. (1970) XI.6.1-6. 